\title{
Sicherheit und Wirksamkeit auf dem Prüfstand
}

Fragestellung: Die Studie prüfte Umfang und Ausmaß von Sicherheit und Wirksamkeit von Fingolimod in der Behandlung der schubförmigen Multiple Sklerose (MS) über einen Zeitraum von zwei Jahren im Vergleich zu Placebo.

Hintergrund: Fingolimod ist ein Sphingosin-Phosphat-Modulator, der zur Behandlung der schubförmigen MS zugelassen ist. In zwei Phase-III-Studien (FREEDOMS I und TRANSFORMS) hatte Fingolimod bereits zeigen können, dass es in OutcomeParametern wie der jährlichen Schubrate hochsignifikant wirksamer als Placebo ist (FREEDOMS I) und auch im direkten Vergleich mit Interferon-Präparaten (TRANSFORMS) überlegen ist.

FREEDOMS II ist die dritte große Phase-III-Studie, in der Fingolimod gegen Placebo verglichen wurde.

Patienten und Methodik: FREEDOMS II ist eine placebokontrollierte, randomisierte doppelblinde, dreiarmige Phase-IIIStudie, die in 117 Zentren, überwiegend in den USA, durchgeführt wurde. Eingeschlossen werden konnten Patienten mit einer schubförmig verlaufenden MS im Alter zwischen 18 und 55 Jahren. Die Patienten wurden zu Fingolimod 0,5 mg oder Fingolimod 1,25 mg oder Placebo randomisiert. Der

Calabresi PA, Radue EW, Goodin D et al. Safety and efficacy of fingolimod in patients with relapsing-remitting multiple sclerosis (FREEDOMS II): a double-blind, randomized, placebo-controlled, phase 3 trial. Lancet Neurol 2014; 13: 545 - 56 primäre Studienendpunkt war die jährliche Schubrate im Monat 24. Sekundäre Endpunkte waren die Zeit bis zur klinisch bestätigten Progression oder die prozentuale Änderung des Hirnvolumens.
Ergebnisse: In die FREEDOMS-II-Studie wurden insgesamt 1.083 Patienten mit schubförmiger MS in den Jahren 2006 bis 2009 eingeschlossen. Davon erhielten 370 Patienten Fingolimod 1,25 mg und 358 Patienten Fingolimod 0,5 mg sowie 355 Patienten Placebo. Die mittlere jährliche Schubrate betrug unter Placebo 0,4, unter Fingolimod 1,25 mg 0,20 (- 50\%) und unter Fingolimod 0,5 mg 0,21 (-48\%). Damit wiesen beide FingolimodGruppen hoch signifikant niedrigere Schubraten auf als die Placebogruppe.

Während sich bei der bestätigten Behinderungsprogression nach 24 Monaten kein signifikanter Unterschied zwischen den Gruppen zeigte (unter Fingolimod 1,25 mg waren 78,3\% der Patienten ohne Behinderungsprogression, unter Fingolimod 0,5 mg 74,7 \% und unter Placebo $71 \%$ ), war die Entwicklung der Hirnatrophie signifikant unterschiedlich. So betrug die Abnahme des mittleren Hirnvolumens nach 24 Monaten unter Fingolimod 1,25 mg - 0,59\%, unter Fingolimod 0,5 mg - 0,85\% und unter Placebo - $1,28 \%$.

Die wichtigsten und häufigsten Nebenwirkungen waren unter Fingolimod 0,5 mg Lymphopenien ( $8 \%$ vs. $0 \%$ unter Placebo), Leberenzymerhöhungen ( $8 \%$ vs. $2 \%$ unter Placebo), HerpesZoster-Infektionen (3\% vs. $1 \%$ unter Placebo) und AV-Block ersten Grades (5\% vs. $2 \%$ unter Placebo). An schweren Nebenwirkungen traten Basalzellkarzinome (3\% vs. $1 \%$ unter Placebo), Makulaödeme (1\% vs. $1 \%$ unter Placebo) und andere Neoplasien ( $4 \%$ vs. $2 \%$ unter Placebo) auf.

Schlussfolgerungen: Auch die dritte Phase-III-Studie zu Fingolimod belegt die überzeugende Wirksamkeit der Substanz in der Behandlung der schubförmigen Verlaufsform der MS und bestätigt darüber hinaus das gute Nebenwirkungsprofil von Fingolimod.

\section{- Kommentar von Volker Limmroth, Köln-Merheim}

\section{Im Westen nichts Neues: Wirksamkeits- und Sicherheitsdaten bestätigt}

Drei Jahre nach Zulassung von Fingolimod kommen nun die Daten der dritten Phase-III-Studie, und nach FREEDOMS I der zweiten umfangreichen Studie, in der Fingolimod gegen Placebo untersucht wurde. Die Daten sind keine Überraschung und replizieren im wesentlichen die Daten, die wir bereits aus FREEDOMS I kennen. Das ist beruhigend, denn sowohl die Wirksamkeits- als auch die Sicherheitsdaten sind fast identisch.

Schaut man etwas tiefer in die Details der Ergebnisse zur Wirksamkeit reduziert Fingolimod signifikant die jährliche Schubrate um $50 \%$, während sich die Entwicklung der Erkrankungsprogression zwischen Fingolimod und Placebo nach 24 Monaten zwar im Trend zugunsten von Fingolimod, aber eben nicht signifikant unterscheidet.

Signifikant unterschiedlich ist jedoch die Entwicklung der Hirnatrophie, die unter Placebo signifikant schneller voranschreitet als in den beiden Fingolimod-Gruppen. Damit zeigt sich in FREEDOMS II erneut, dass der EDSS als Outcome-Parameter bei Patienten mit relativ geringer Erkrankungsdynamik (Baseline-EDSS ca. 2,5) sehr träge und die Messung der Hirnatrophie bei dieser Patientengruppe offensichtlich sensitiver ist.

Auch das Nebenwirkungsspektrum entsprach dem bereits gut bekannten: Lymphopenien, leichte Leberenzymerhöhungen, Herpes-Zoster-Infektionen, Erstdosis-Bradykardien, AVBlock, und alles jeweils in milden bis moderaten Ausmaßen, die nur selten zum Absetzen der Medikation zwangen.

Mit den nun aus drei umfangreichen Phase-III-Studien zur Verfügung stehenden Sicherheitsdaten ist die von der EMA 2011 erteilte Zulassung als Secondline-Therapie eigentlich nicht mehr notwendig oder zu rechtfertigen. Eine Firstline-Zulassung wie in den USA, der Schweiz, Australien und vielen anderen Ländern ist jetzt eigentlich überfällig und würde die Therapie von MS-Patienten erleichtern. 